\title{
Immunopharmacology of fatty acids
}

Philip C. Calder ${ }^{1,2}$ and Linette E.M. Willemsen ${ }^{3}$

${ }^{1}$ Faculty of Medicine, University of Southampton, Southampton, United Kingdom

${ }^{2}$ NIHR Southampton Biomedical Research Centre, University Hospital Southampton NHS

Foundation Trust and University of Southampton, Southampton, United Kingdom

${ }^{3}$ Division of Pharmacology, Utrecht Institute for Pharmaceutical Sciences, Faculty of

Science, Utrecht University, Utrecht, The Netherlands

Fatty acids have several physiological roles. They are important energy sources, including for cells of the immune system, and they are vital membrane constituents, where they are incorporated into several different complex lipids including phospholipids. The fluid mosaic model of cell membranes recognised that cell membrane lipids form a fluid environment within which proteins "float" and that this environment is important for the function of those proteins, which are often involved in signalling processes. The fatty acid composition of membrane lipids is an important determinant of membrane "fluidity". The recognition of the role of lipids, including their fatty acid constituents, within membranes has been further advanced over the last fifteen years or so with the discovery of membrane microdomains termed rafts. These are regions of membranes with a distinct, characteristic structural composition that act as platforms to colocalise proteins involved in intracellular signalling pathways. Rafts are particularly important in cells of the immune system because those cells are highly responsive to extracellular signals. Fatty acids seem to have roles in promoting or discouraging the formation of lipid rafts, including in cells of the immune system, and hence can affect intracellular signalling through that mechanism. Related to this, many lipid structures that act as signalling molecules, including phospholipids, lysophospholipids, diacylglycerols, sphingolipids and endocannabinoids, include fatty acids in their structure and altering the fatty acid makeup alters the effect and/or the potency of the signalling lipid. Thus, changing the fatty acid composition of the complex lipids within an immune cell membrane can affect the latter's physical properties, the ability of the membrane to form signalling platforms, and the activity of membrane lipid-derived signalling molecules. A further role for membrane fatty acids is to act as the substrate for the formation of bioactive oxidised derivatives. Among the most well known of these derivatives are the prostaglandins, 
thromboxanes and leukotrienes. The 2 and 4 series of these are formed from the omega- 6 fatty acid arachidonic acid made available from membrane phospholipids. These mediators are converted enzymatically by cyclooxygenase, lipoxygenase and cytochrome P450 enzymes. Cells involved in the immune response are typically important producers of these mediators, which are also potent regulators of the activity of T-cells, B-cells, monocytes, macrophages, granulocytes and many other cell types. These mediators are frequently overproduced in diseases involving an excessive inflammatory response and several common anti-inflammatory drugs target the enzymes involved in their biosynthesis. Omega-3 fatty acids compete with omega- 6 fatty acids for membrane incorporation and are also released from the membrane. Eicosapentaenoic acid is precursor for less inflammatory 3 and 5 series of eicosanoids and competes with arachidonic acid to be converted by cyclooxygenase, lipoxygenase and P450 enzymes. One of the most exciting discoveries in this field in recent times has been of novel families of mediators derived from eicosapentaenoic acid and docosahexaenoic acid omega-3 fatty acids, including the resolvins and protectins, which have potent activity in the resolution of inflammation.

Each of the mechanisms linking fatty acids to (immune) cell function described above involve fatty acids being chemically bound into complex membrane lipids like phospholipids. However, mechanisms of fatty acid action within the immune system involving binding of non-esterified (i.e., “free”) fatty acids to cell surface receptors including the G-protein coupled receptors and some of the toll-like receptors have been described. In particular the role of docosahexaenoic acid has been exemplified, since it can modulate intracellular signalling by binding G-protein coupled receptor 120 and it forms a complex with peroxisome proliferator-activated receptor- $\gamma$ modifying gene transcription. These mechanisms suggest that fatty acids do not need to be bound into complex membrane lipids to have an effect on immune cell function and that fatty acids may act within the immune system as ligands binding to cell surface, and intracellular receptors.

There is currently much research interest in the effects of fatty acids, and more complex lipids that include fatty acids in their structure, on immune cell functional responses. This area of research has clear public health and clinical applications. However, this is not a new area of research: the first studies of fatty acids and immune cell function were published in the 1970s and the first comprehensive review of the field was published by Meade and 
Mertin in 1978. Nevertheless, the plethora of possible interlinked mechanisms involved, many of which have been discovered recently; the sophisticated molecular, cellular and immunologic techniques now available for research. Furthermore the increasing recognition of the role of a dysregulated immune response in human disease combine to make this a very exciting and topical field, that has advanced significantly in a relatively short period of time. In this Special Issue of European Journal of Pharmacology a number of experts provide state-of-the-art literature reviews of research areas relevant to this topic. These reviews cover a number of fatty acids, several complex lipids, and an array of bioactive mediators derived from fatty acids. They provide new insights into the mechanisms of action described above. Finally, the reviews discuss possible therapeutic roles for fatty acids and their derivatives in conditions including inflammation of the airways, the gut mucosa, the joints, the central nervous system and metabolic tissues. Together, these reviews provide a superb collation of the current state-of-the-art in the field, they serve to highlight the most exciting developments in the field, and clearly indicate its importance to better understanding human health and disease. We think that this collection of reviews will be a valuable resource for readers. 